\title{
Research Paper \\ Predicting the Intensity of Pain in Patients With Chronic Pain Based on Alexithymia: The Mediating Role of the Behavioral Inhibition System
}

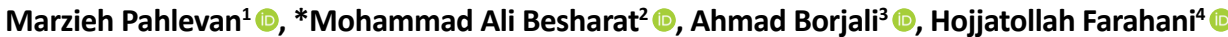

1. PhD. Candidate in Health Psychology, Department of Psychology, Faculty of Psychology, Karaj Branch, Islamic Azad University, Karaj, Iran. 2. PhD. in Clinical Psychology, Professor, Department of Psychology, Faculty of Psychology and Education, University of Tehran, Tehran, Iran.

3. PhD. in Psychology, Associate Professor, Department of Clinical Psychology, Faculty of Psychology and Educational Sciences, Allameh Tabatabaee University, Tehran, Iran 4. PhD. in Psychometrics, Assistant Professor, Department of Psychology, Faculty of Humanities, Tarbiat Modares University, Tehran, Iran.

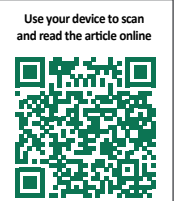

cittation Pahlevan M, Besharat MA, Borjali A, Farahani H. [Predicting the Intensity of Pain in Patients With Chronic Pain Based on Alexithymia: The Mediating Role of the Behavioral Inhibition System (Persian)]. Iranian Journal of Psychiatry and Clinical Psychology. 2019; 25(1):56-71. http://dx.doi.org/10.32598/ijpcp.25.1.56

doi) $h$ http://dx.doi.org/10.32598/ijpcp.25.1.56

Received: 13 Jan 2018

Accepted: 17 Sep 2018

Available Online: 01 Apr 2019

Keywords:

Chronic pain, Emotion, Brain, Behavior

\section{A B S T R A C T}

Objectives This study aimed to predict the intensity of pain based on the amount of alexithymia in patients with chronic pain, and in this relationship, the behavioral inhibition system has a mediating role. Methods The present study has a correlational design and carried out with a structural equation modeling approach. The statistical population comprised all 20-60 years old patients with chronic pain who had referred to Mahan Clinic and the physical medicine and rehabilitation clinic of Arman in Tehran, from spring to autumn of 2017. Of them, 488 patients who had experienced the musculoskeletal pain for at least 3 months, were chosen purposefully and asked to respond the Toronto Alexithymia Scale (TAS-20), the inhibition/activation system scale, and Numeric Rating Scale (NRS). The analysis was done in SmartPLS.

Results The intensity of pain had a positive relationship with inhibition system $(P<0.001)$ and fight/ flight system $(P<0.05)$. Alexithymia had the positive a relationship with inhibition system $(P<0.001)$. Alexithymia ( $t=6.34, \beta=0.29)$, inhibition system ( $t=3.77, \beta=0.17)$, and fight/ flight system ( $t=4.26, \beta=0.18)$ could explain the variance of the pain intensity. Alexithymia could explain the activity of inhibition system $(t=8.03, \beta=0.30)$ and the behavioral activation system $(t=2.83, \beta=-0.14)$.

Conclusion The relationship between alexithymia and the intensity of pain was not a simple direct relationship, but the inhibition system with its avoidance behavior output could affect this relationship.

\section{Extended Abstract}

\section{Introduction}

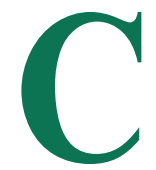

hronic pain refers to a continuous pain lasted for at least 3 months. It is common among adults and causes problems for an individual's health and the quality of life. According to studies, the patient with chronic pain perceives their pain with a higher intensity. One of the most important factors that affects the severity and duration of pain is alexithymia. People with alexithymia cannot understand their emotions well, struggle to express their emotions to others, and their thinking is operational. So, they externalize their emotion with physical signs like chronic pain. This externalization is not only the result of their personality but has some physical root, too.

a

* Corresponding Author:

Mohammad Ali Besharat, PhD.

Address: Department of Psychology, Faculty of Psychology and Education, University of Tehran, Tehran, Iran.

Tel: +98 (21) 61117488

E-mail: besharat@ut.ac.ir 
Many studies indicate that these people express a lot of avoidance in their actions, but no study has studied the role of brain behavioral systems, especially the behavioral inhibition system in this phenomenon. We postulate in our study that behavioral inhibition system has a mediating role in the relationship of chronic pain with alexithymia and that the avoidance behavior in patients with chronic pain is not just a response to the pain, but it may have a significant role in their brain behavioral systems.

\section{Method}

The present study design was correlational and was carried out with a structural equation modeling approach. The statistical population was all 20-60 years old patients with chronic pain who had referred to the Mahan Clinic and the physical medicine and rehabilitation clinic of Arman in Tehran, from spring to autumn of 2017. Out of them, 488 patients ( 258 women, and 182 men) who reported the chronic musculoskeletal pain for at least 3 months, were chosen purposefully. They were asked to complete the study questionnaires. The analysis was done in SmartPLS software.

The first study tool was Numeric Rating Scale (NRS). It is a visual rating scale for assessing the intensity of pain by asking the patients to choose a number between 0 and 10 for the intensity of their pain. The second study tool was Toronto Alexithymia Scale (TAS-20). It has 20 questions and scored on a 5-point Likert-type scale, from 1 (Completely disagree) to 5 (Completely agree). The scale reveals three factors: difficulty in identifying feelings, difficulty in describing feelings, and externally-oriented thinking. The reliability and validity of the Persian version of this scale have been assessed by Besharat [48].

The psychometric properties of this scale were confirmed in that research. The third study tool was the inhibition/activation system scale. It assesses the Behavioral Activation
System (BAS) and the Behavioral Inhibition System (BIS). The scale has 24 items and is scored based on a 4-point Likert-type scale (from 1 to 4). Factor analysis confirmed the psychometric properties of the scale. In Iran, the psychometric properties of the Persian version of this scale were also assessed on different groups and confirmed, too. Following the Heim et al. study [50], we used this scale for three subscales instead of two, after doing a factor analysis and finding a group of questions in accordance with Fight/ Flight System (FFS).

\section{Results}

Table 1 presents the correlations of the study variables. The model was assessed with SmartPLS because we had a variable (intensity of pain) with one question. To assess the reliability of the model, using Cronbach alpha, communality, and the composite reliability was appropriate. To assess the validity of the model, we eliminated the questions with factor load below or near to 0.7 based on Churchill approach [55]. The Average Variance Extracted (AVE) was more than 0.5 , and also less than the composite reliability. The crossloading test and Fornell-Larcker test for divergent validity were appropriate, too. Figure 1 displays the model. Table 2 presents the test of the significance of the path shown.

To illustrate the moderating role of brain behavioral system, we used the Sobel test that proved the moderating role, just for BIS, between alexithymia and pain intensity ( $\mathrm{Zs}=3.416, \beta=0.049, \mathrm{P}=0.000)$. We used the Goodness of Fit (GOF) to assess the quality of the model. The result was $\mathrm{GOF}=\sqrt{\text { average }}$ (communality)* $\mathrm{R}^{\wedge} 2=0.400$. This quality compared with three values $(0.19,0.33,0.67)$ set by Chin [55], was rated upper than average.

\section{Discussion}

The results of this research showed that the relation between alexithymia and pain intensity is positive and signifi-

Table 1. The correlation of the study variables

\begin{tabular}{|c|c|c|c|c|c|}
\hline Variable & 1 & 2 & 3 & 4 & 5 \\
\hline Intensity of pain & 1 & & & & \\
\hline Alexithymia & $0.361^{*}$ & 1 & & & \\
\hline Behavioral inhibition & $0.275^{*}$ & $0.249 *$ & 1 & & \\
\hline Fight/flight system & $0.116^{* *}$ & 0.034 & 0.048 & 1 & \\
\hline Behavioral activation & 0.001 & -0.002 & $0.167^{*}$ & $0.205^{*}$ & 1 \\
\hline
\end{tabular}

* Significance at 0.01 level; ** Significance at 0.05 level. 
Table 2. The significance of the path and hypothesis of the study

\begin{tabular}{ccccc}
\hline Path & T-Test & $\boldsymbol{\beta}$ & SE & Sig. \\
\hline Alexithymia -> BIS & 8.039 & 0.300 & 0.037 & 0.01 \\
\hline Alexithymia -> BAS & 2.839 & -0.140 & 0.049 & 0.01 \\
\hline Alexithymia -> FFS & 0.520 & -0.026 & 0.050 & 0.01 \\
\hline Alexithymia - intensity of pain & 6.348 & 0.290 & 0.045 & 0.01 \\
\hline BIS - intensity of pain & 3.774 & 0.170 & 0.045 & - \\
\hline BAS -> intensity of pain & 0.932 & -0.048 & 0.051 & 0.01 \\
\hline FFS -> intensity of pain & 4.268 & 0.180 & 0.042 & Iranian Journal of
\end{tabular}

cant. It also confirms the first hypothesis of the study and is consistent with previous studies results [2-5]. The similar results might be explained as follows: the patients with alexithymia may have hypersensitivity to physical sensations and express long-term responses to pain because of some deficit in their emotion regulation system. This may be a mechanism that connects the alexithymia to chronic pain [19]. Also, the defect in the ability of the symbolization of emotions may cause problems such as abnormal physical reactions and disability in self-control and self-regulation [7]. This may also affect the intensity and duration of the pain that is comprehended.
The results of this study indicate that the behavioral inhibition system has a mediator role between alexithymia and the intensity of pain that is perceived by the people as chronic pain. Considering the partial role of this mediator, we can conclude that some part of the relationship between alexithymia and the intensity of pain, is mediated through behavior inhibition system. There is also this probability that the neural machinery in alexithymia is activated more at the physiological level, and less at the cognitive-experiential domains of the emotional response system [19]. Briefly, alexithymia as a disability to recognize and state the emotions as well as a tendency to think objectively can increase the activity of inhibition system, resulting in expression of some kind of avoidance or inhibition behavior.

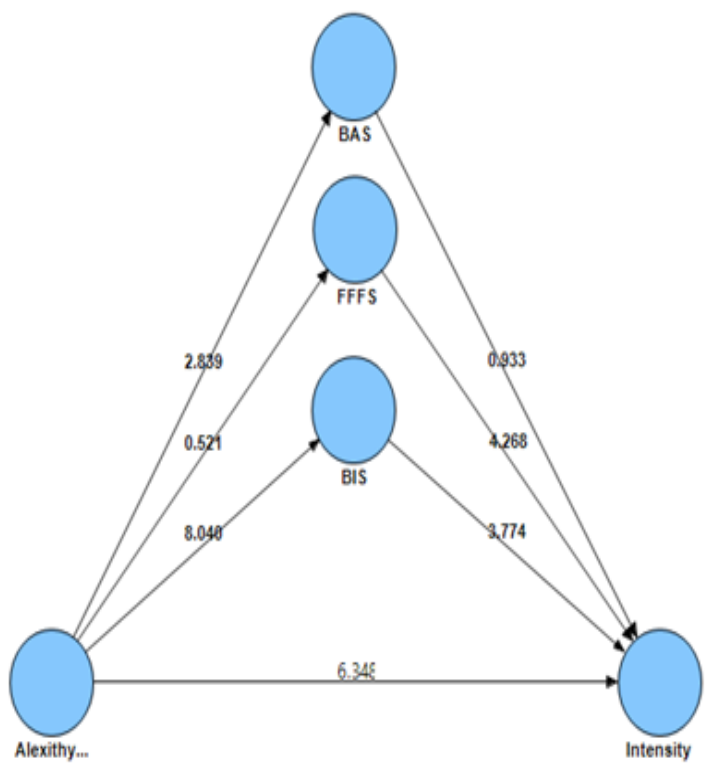

Figure 1. The image of the model in Table 2 presenting the test of the significance of the path 
Whenever the alexithymia is accompanied by the increase of activity in the behavioral inhibition system, the chronic pain is felt with more intensity.

\section{Ethical Considerations}

\section{Compliance with ethical guidelines}

In this research, the form of ethical considerations was filled by the patients and was followed by the researchers.

\section{Funding}

This research did not receive any specific grant from funding agencies in the public, commercial, or not-forprofit sectors.

\section{Authors contributions}

All authors contributed in preparing this article.

\section{Conflict of interest}

The authors declared no conflict of interest.

\section{Acknowledgements}

We would like to acknowledge the staff of Mahan Clinic and The Physical Medicine and Rehabilitation Clinic of Arman, for their cooperation in this study. 
This Page Intentionally Left Blank 


\title{
ييشبينى شدت درد در بيماران مبتلابه درد مزمن بر اساس ناتويى هيجانى: نقش واسطداى سيستم

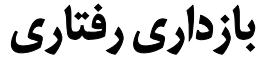

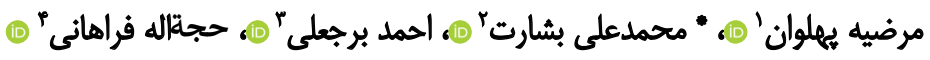

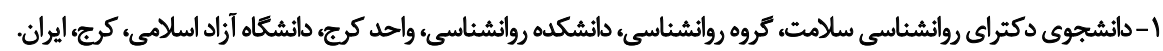

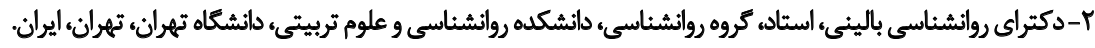

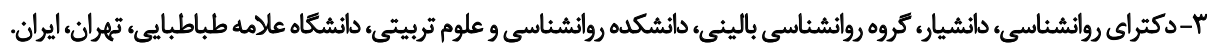

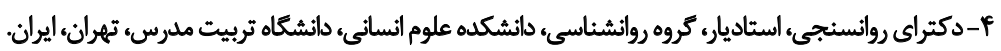

\begin{abstract}
حكيد

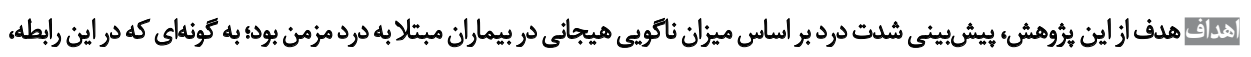

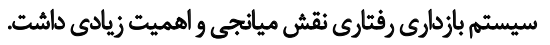

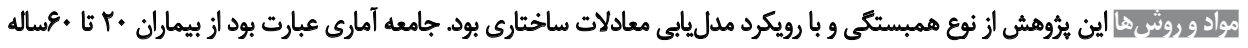

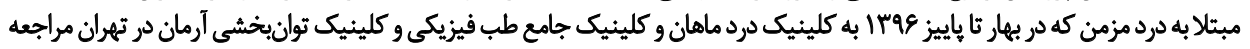

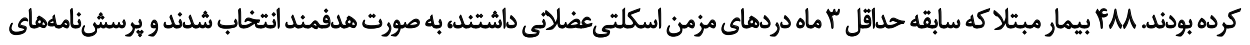

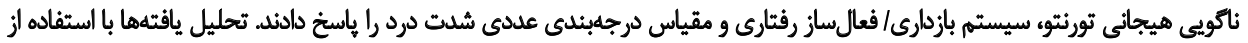

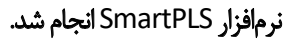

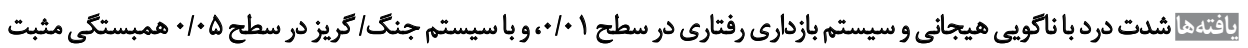

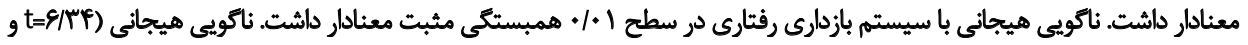

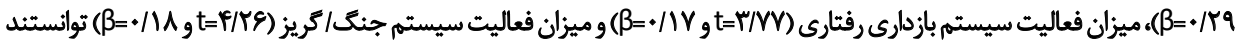

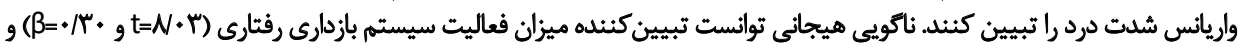

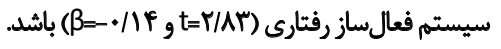

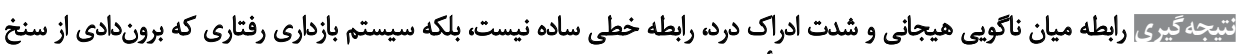

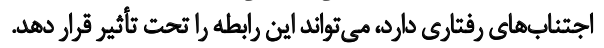

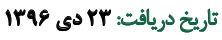

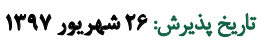
تاريخ انتشار: rا فروردين

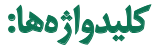

درد مزمن، هيجان، مغز، رفتار

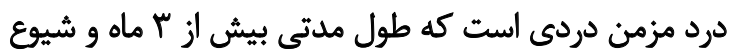

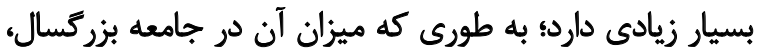

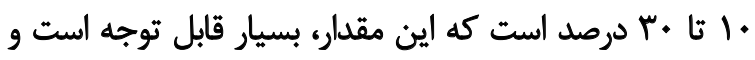

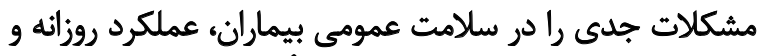

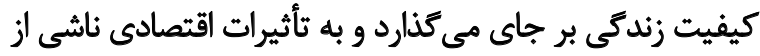

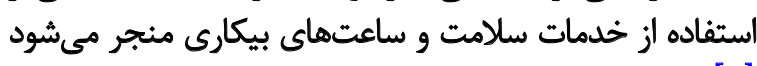

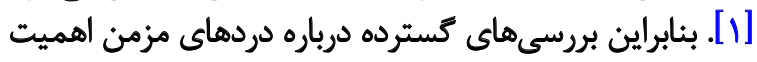

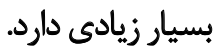

درد مزمن'، يكى از اساسىترين بيمارىهاى روانتنى باست كه

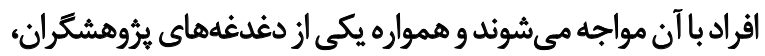

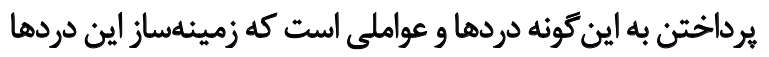

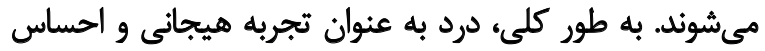
ناخوشايندى تعريف شده است كه با آسيب بافتى واقعى يا با بالقوه همراه است و يا بر اساس جنين تآسيبى توصيف مى آشود.

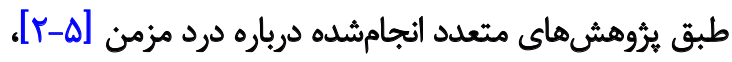

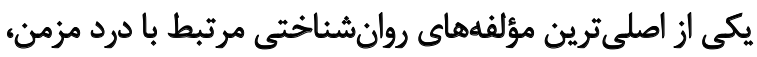


محركهاي مرتبط با اطلاعات فيزيكى در مناطق حسىتنى و

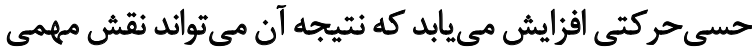

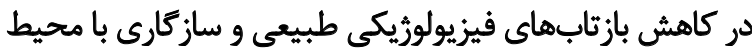

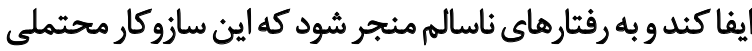

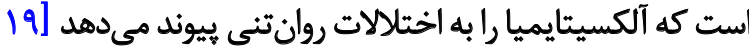

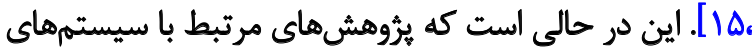

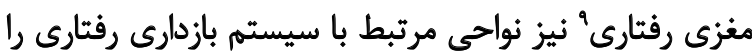

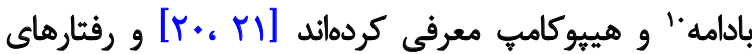
اجتنابى رابا تغيير در ميزان فعاليت اين نواحى مرتبط دانستهاند. از آنجا كه يكى از دغدغههاى يُروهشكران، مسئله تأثيركذارى

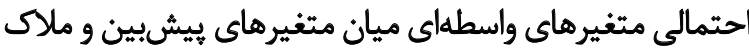

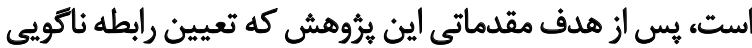

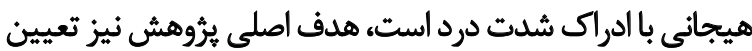

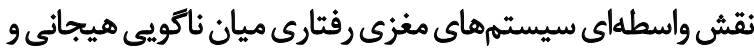
شدت درد ادراكشده در بيماران مبتلا به درد مزمن است. سيستمهاى مغزى رفتارى كه برآمده از نظريه حساسيت

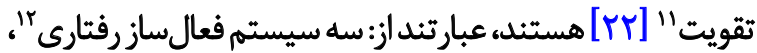

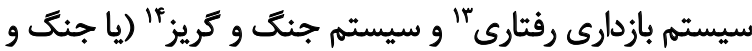

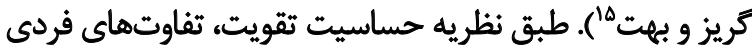

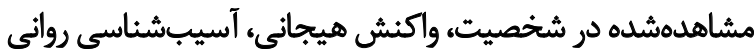

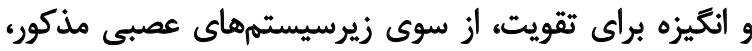

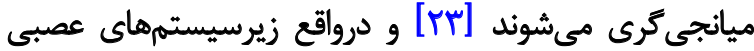

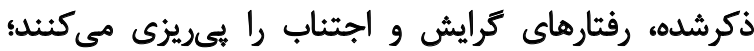

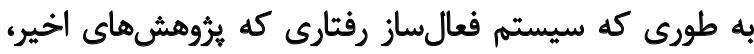

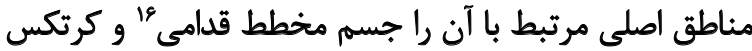

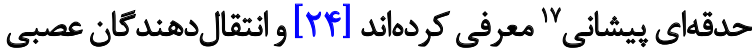

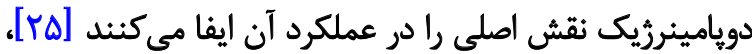

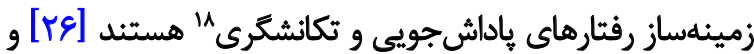

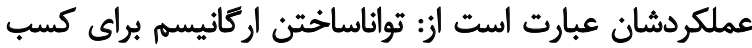

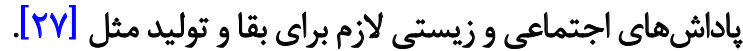

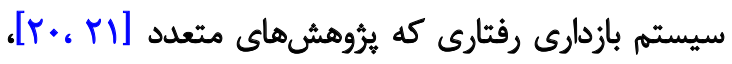

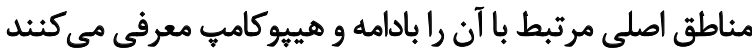

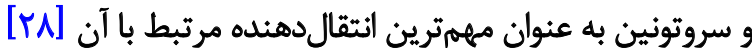

9. Brain behavioral systems

10. Amygdala

11. Reinforcement Sensitivity Theory (RST)

12. Behavior Activation System (BAS)

13. Behavior Inhibition System (BIS)

14. Fight, Flight System (FFS)

15. Fight, Flight, Freeze System (FFFS)

16. Ventral striatum

17. Orbito frontal cortex

18. Impulsivity

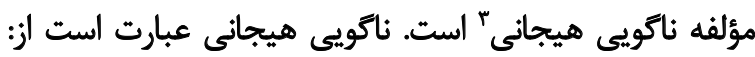

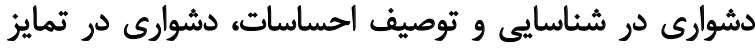

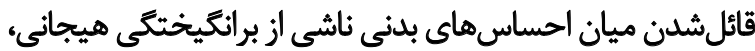

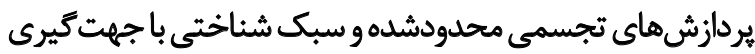

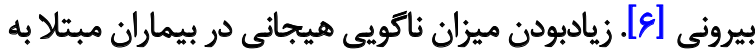

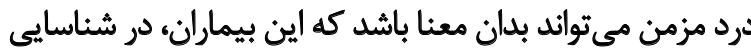

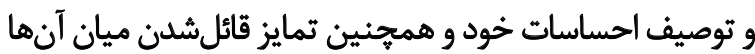

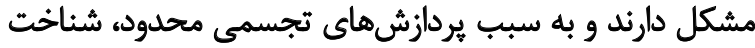

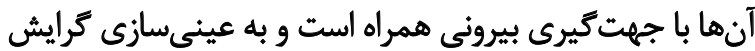

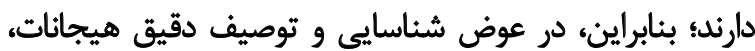

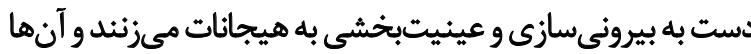

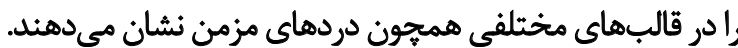

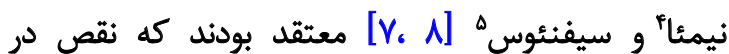

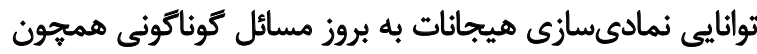

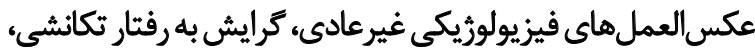
ناراحتى و اجتناب از ارتباطات اجتماعى و وتوانيايى آسيبديديديه

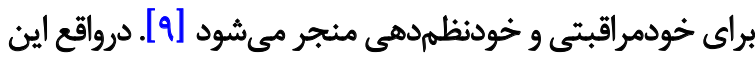

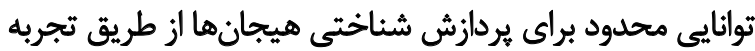

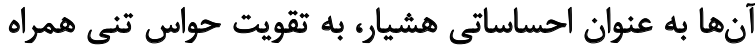

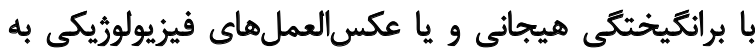

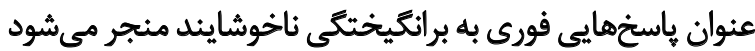

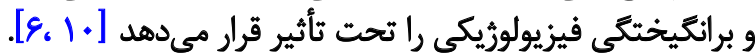

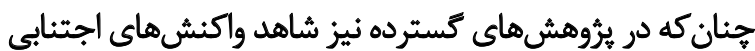

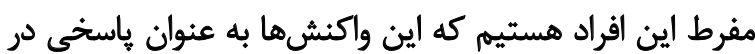

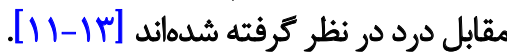

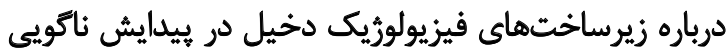

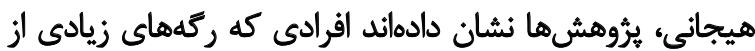

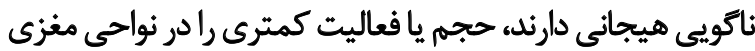

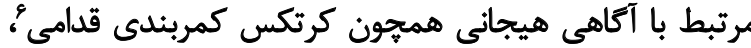

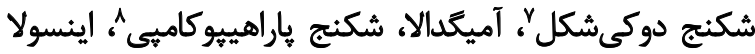

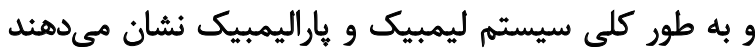

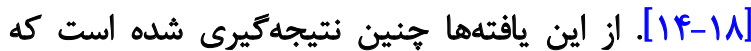

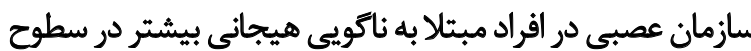

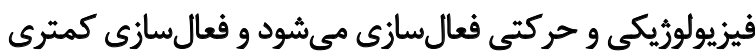

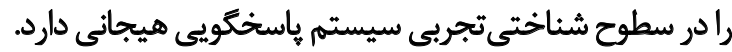
در اين افراد، ياسخكويى عصبى به محرك هيجانى در سيستم

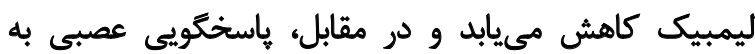

3. Alexithymia

4. Nemiah

5. Sifneos

6. Anterior cingulate cortex

7. Fusiform gyrus

8. Parahyppocampal gyrus 


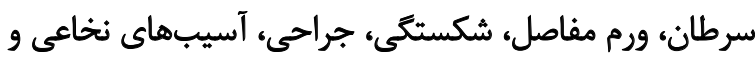

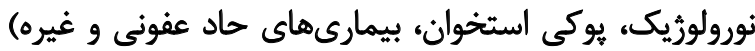

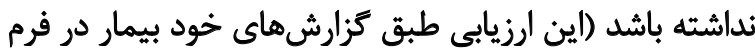

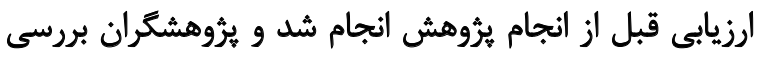

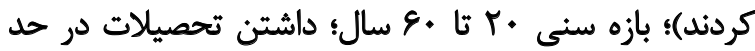

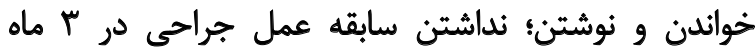

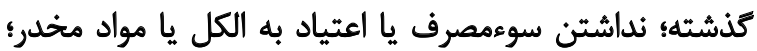

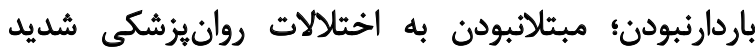

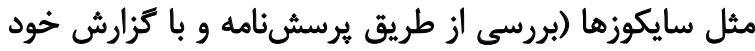

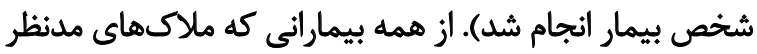

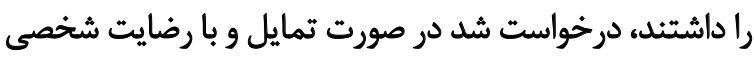

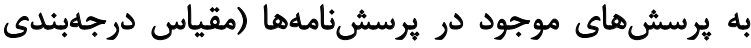

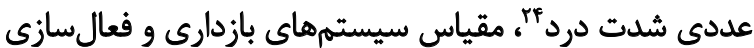

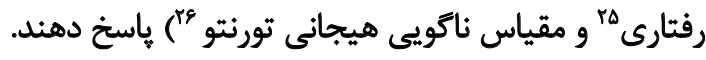

براى درنظر كرفتن نفع و سودمندى يروهش، كتاب راهنمايى

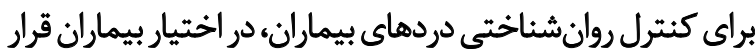

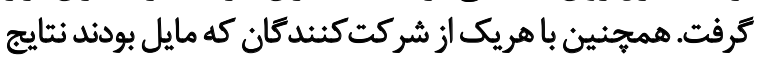

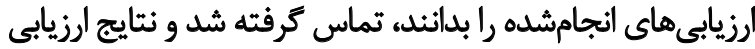

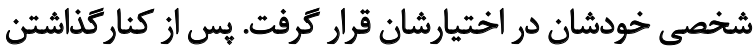

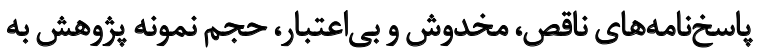
ن نff.

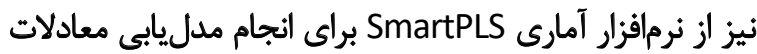

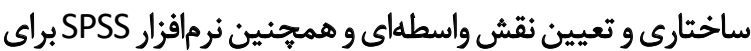
آزمون همبستكى استفاده شد.

\section{ابزار جمعآورى اطلاعات}

\section{مقياس درجهبندى عددى شدت درد}

مقياس درجهبندى عددى شدت درد، محور ديدارى

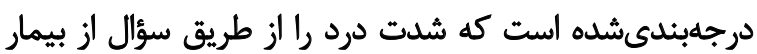

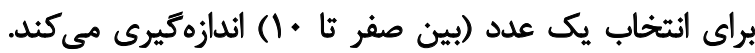

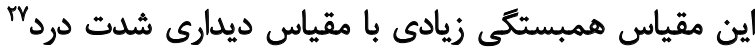

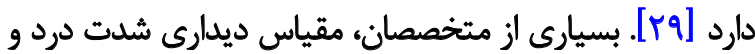

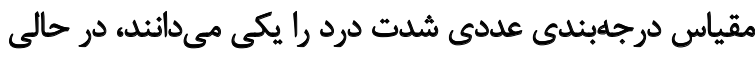

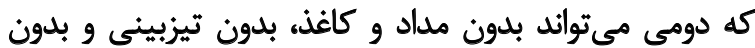

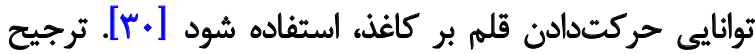

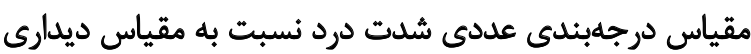

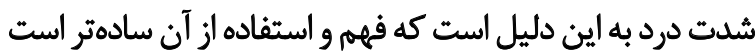

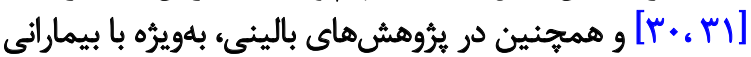

24. Numeric Rating Scale (NRS)

25. Behavioral Activation/Inhibition System (BAS/BIS)

26. Toronto Alexithymia Scale (TAS)

27. Visual Analog Scale (VAS)
در نظر كرفته شده است، وظيفه حل تعارض ميان اهداف

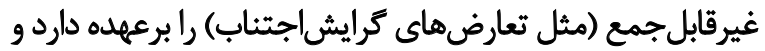

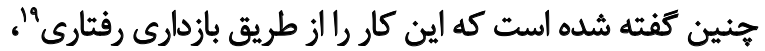

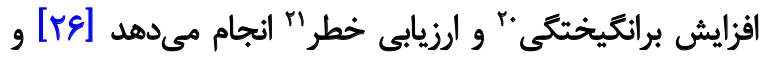

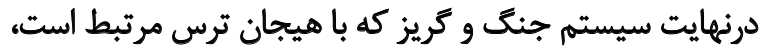

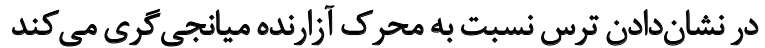

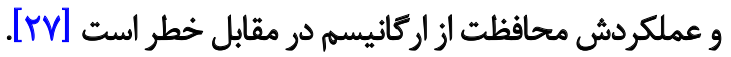

هريك از اين زيرسيستمها كه زمينههائ زيستى كوناكون دارئد،

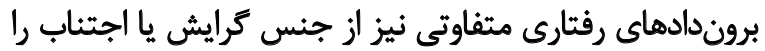

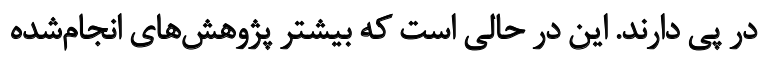

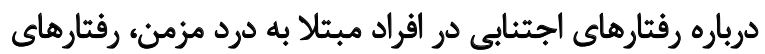

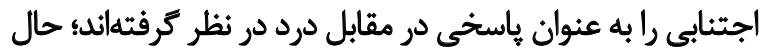

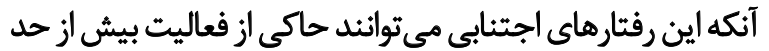

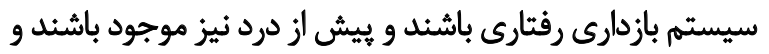

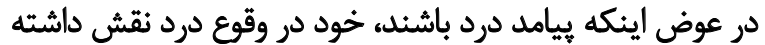

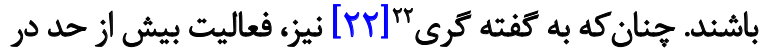

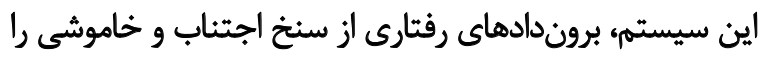
به همراه خواهد داشت بن برون

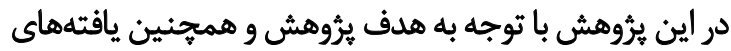

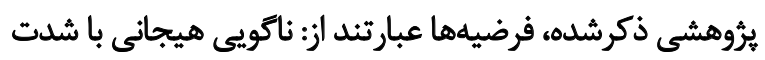

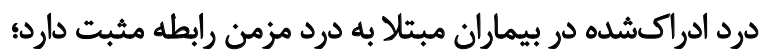

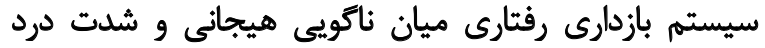

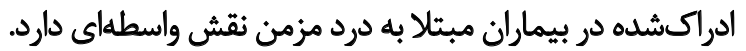

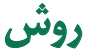

اين يُوهش از نوع همبستكى و با رويكرد مدل يابى معادلات

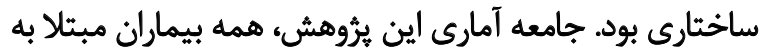

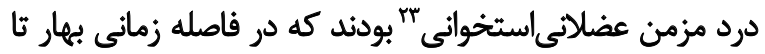

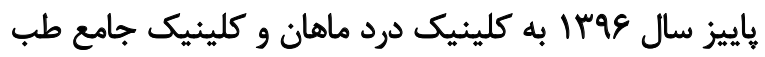

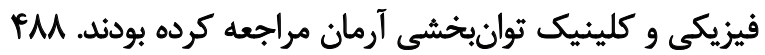

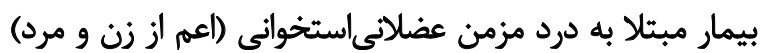

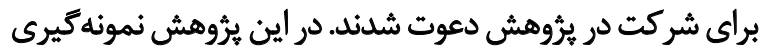

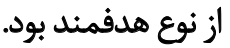

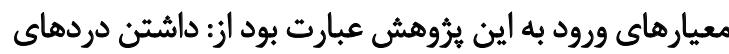

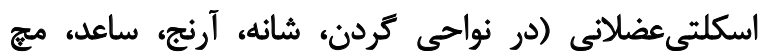

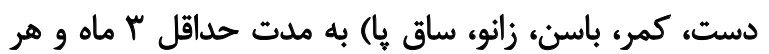

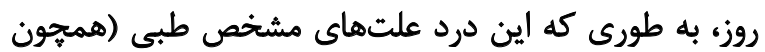

19. Inhibiting behavior

20. Increasing arousal

21. Assessing for risk

22. Gray

23. Chronic musculoskeletal pain 
ياداش از سيستم فعالساز رفتارى نيز وعا • كزارش شده است. ويزَٔىهاى روانسنجى اين يرسشنامه در ايران را محمدي،

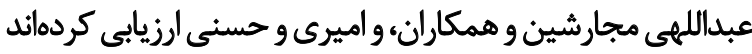

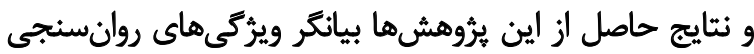

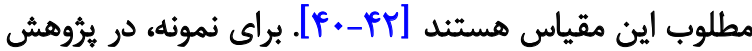

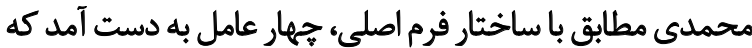

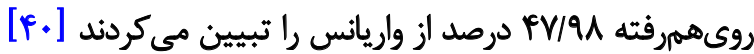

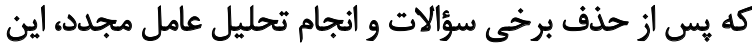
مقدار به هQ/ه درصد افزايش يافت.

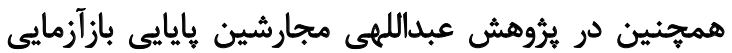

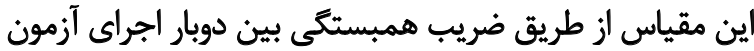

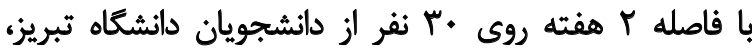

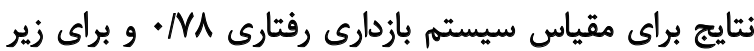

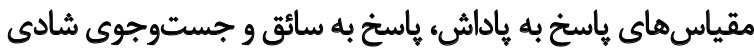

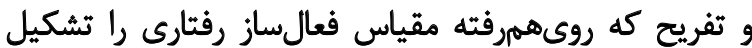

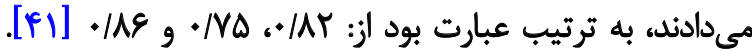

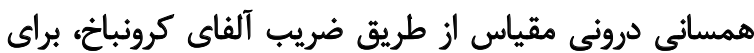

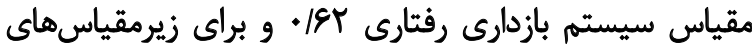

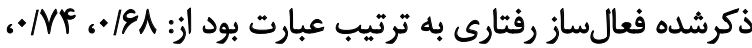

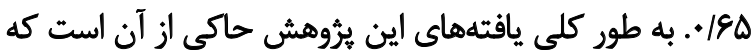

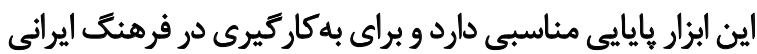

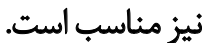

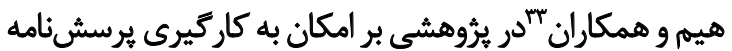

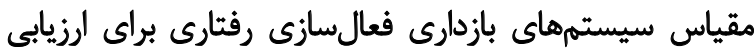

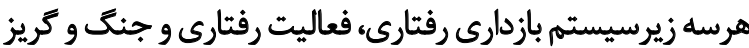

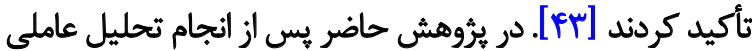

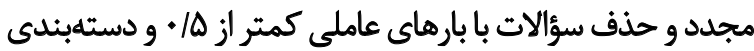

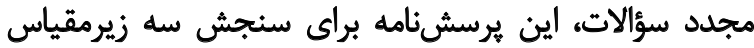

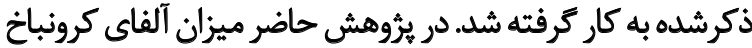

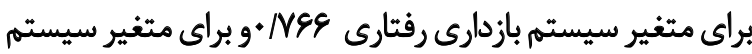

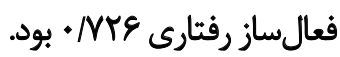

به طور كلى يافتههاى يُروهش حاضر حاكى از آن است كه اين

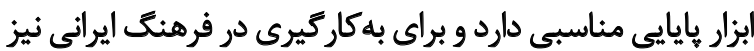

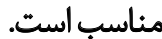

\section{مقياس ناكويى هيجانى تورنتو}

مقياس ناكويى هيجانى تورنتو يك آزمون · T سؤالى است و سه

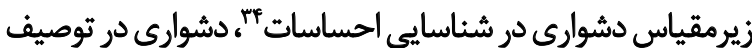

33. Heym

34. Difficulty in identifying feelings
كه تحصيلات كمترى دارنده اعتبار زيادى را نشان مى دهد [بr]]. درباره اين مقياس، اعتبار بازآزمايى زيادى در هر دو درو دروه

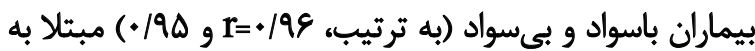

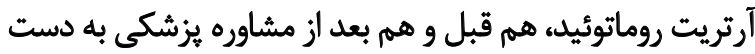

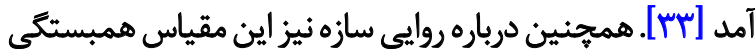

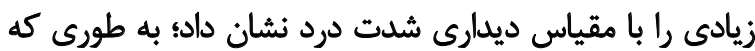

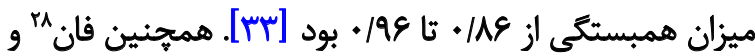

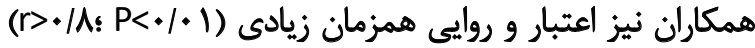

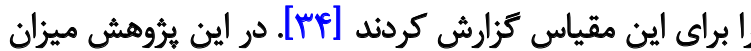

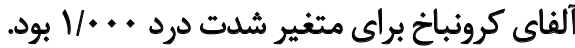

\section{مقياس سيستمهاى بازدارى و فعالسازى رفتارى}

اين مقياس راكارور و وايت بَ براى ارزيابى سيستمهاى بازدارى

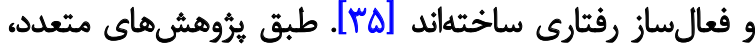

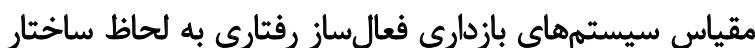

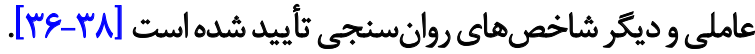

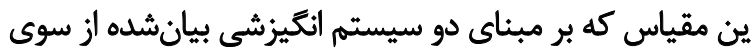

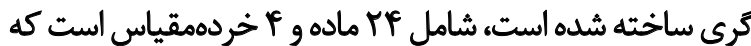

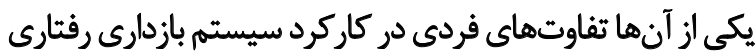

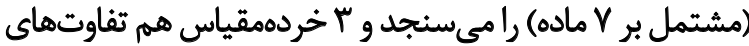

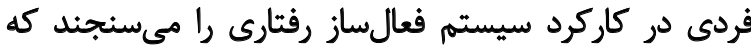

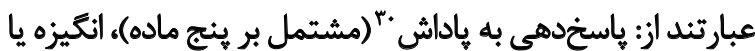

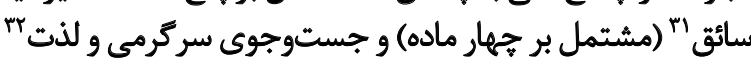

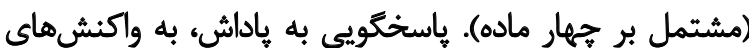

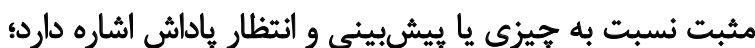

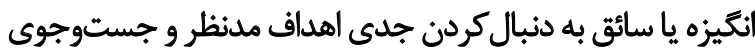

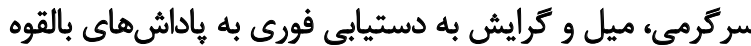

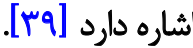

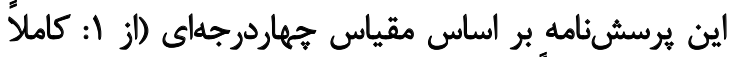

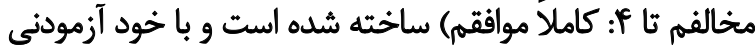

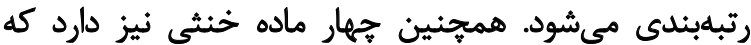

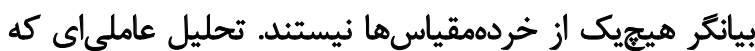

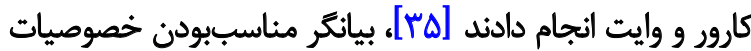

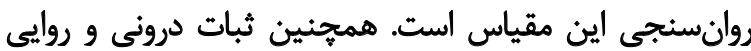

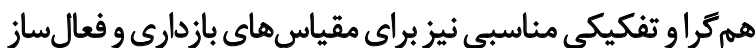

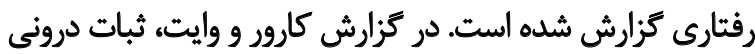

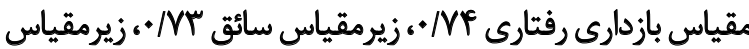

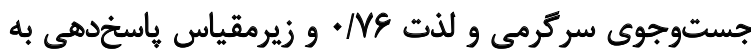

28. Phan

29. Carver \& White

30. BAS reward responsiveness

31. BAS drive

32. BAS fun seeking 


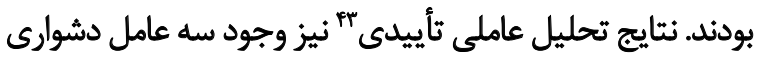

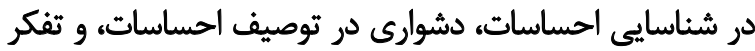

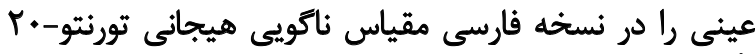

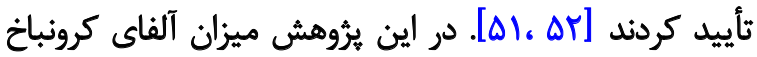

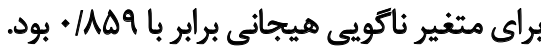

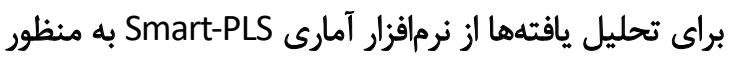

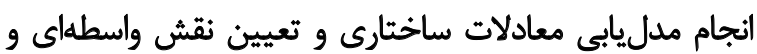
همجنين نرمافزار SPSS براى آزمون همبستئى استفاده شد.

يافتهما

افراد بررسىشده، PI/F)

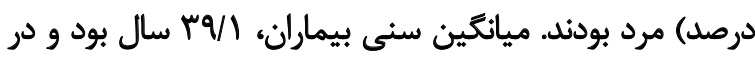

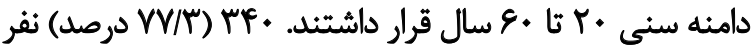

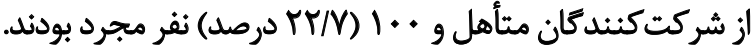

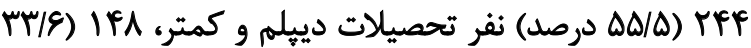

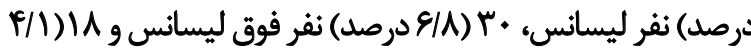
درصد) نفر مدرك تحصيلى دكترا داشتئد.

در اين يُروهش فرضيه اصلى ميزان فعاليت سيستمهاي

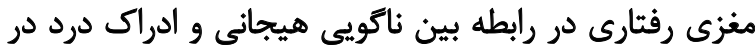

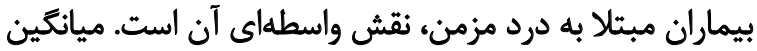

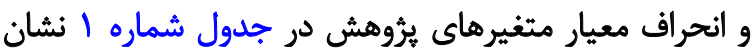
داده شده الست معيت

نتايج آزمون همبستكى بيرسون در جدول شارل شماره ب نشان

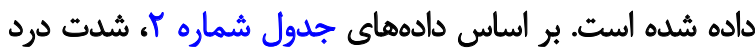

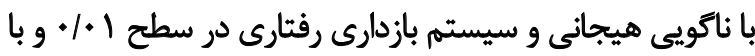

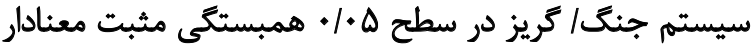

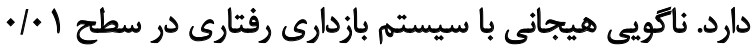

$$
\text { همبستكى مثبت معنادار دارد. }
$$

43. Confirmatory factor analysis

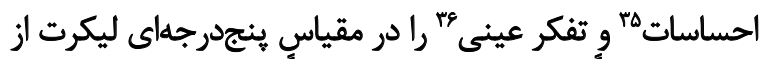

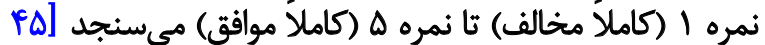
[Ff.

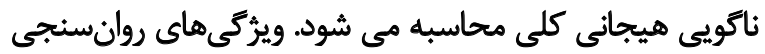

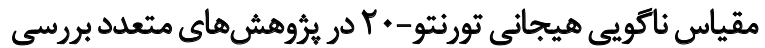

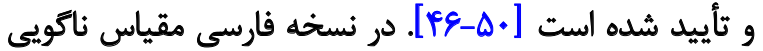

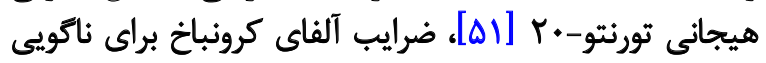

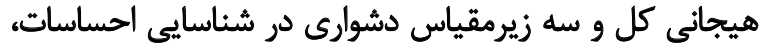

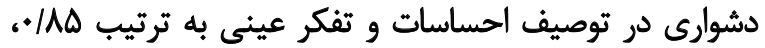

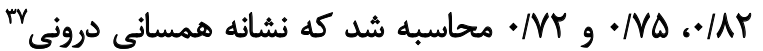

$$
\text { خوب مقياس است. }
$$

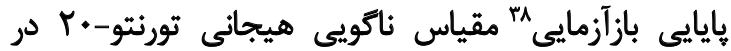

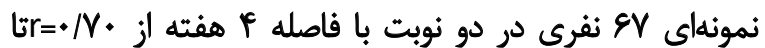

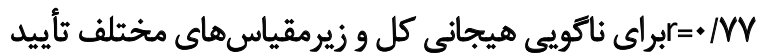

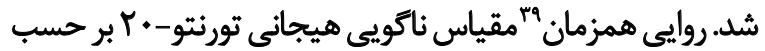

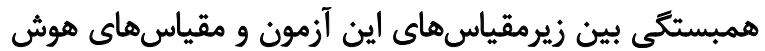

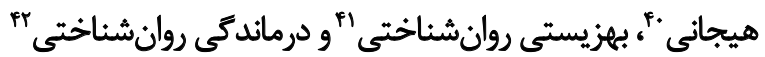

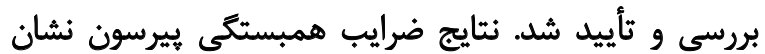

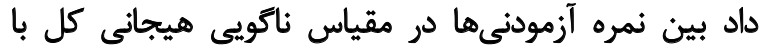

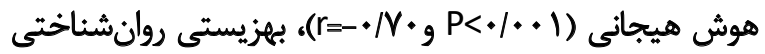
(1)

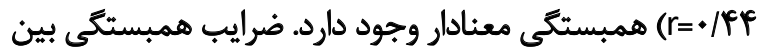

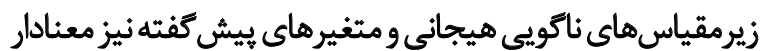

35. Difficulty in describing feelings

36. Externally oriented thinking

37. Internal consistency

38. Test-retest reliability

39. Concurrent validity

40. Eemotional intelligence

41. Psychological well-being

42. Psychological distress

\begin{tabular}{|c|c|c|c|}
\hline \multicolumn{3}{|c|}{ ميانكين土|نحراف معيار } & \multirow{2}{*}{ مثغير } \\
\hline كل & زنان & مردان & \\
\hline$\varepsilon \mid \& \& \pm T / .$. & $V / \circ \Delta \pm V / A V$ & $g / 11 \pm T / .94$ & ش شدت درد \\
\hline$M / M I N A M$ & $M / g N \pm N R$ & $r T / r+ \pm V / a F$ & ناكويى هيجانى \\
\hline$g / \Delta V \pm 1 / \pi r$ & $g / N \pm D / Q$ & $g / r \Delta \pm 1 / r \Lambda$ & سيستم بازدارى رفتارى \\
\hline $1 W / 9 \cdot \pm r / \cdot q$ & 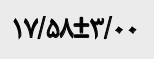 & $W r V \pm r / \cdot 9$ & سيستم فعال ساز رفتارى \\
\hline$\Delta / r \backslash \pm V / \Delta f$ & $\Delta / . Y \pm V / Q Y$ & $\Delta / \leftarrow \wedge \pm 1 / \pi V$ & سيستم جنك وكريز \\
\hline
\end{tabular}

جدول ا. ميانكين و انحراف معيار نمرات شدت درد، ناكويى هيجانى، سيستم بازدارى رفتارى، سيستم فعال ساز رفتّارى، و سيستم جنك و كريز 
جدول Y. ضرايب همبستئى بيرسون بين متغيرهاي يرّوهش

\begin{tabular}{|c|c|c|c|c|c|}
\hline 0 & $\varepsilon$ & $r$ & r & 1 & متغير \\
\hline & & & & 1 & شيلت درد \\
\hline & & & 1 & $. / 18)^{*}$ & ناكوييى هيجاني \\
\hline & & 1 &.$/ r e 9^{*}$ & $+/ T V \Delta^{*}$ & سيستم بازدارى \\
\hline & 1 & $1+$ +1 & $.1 . m$ & ./18** & سيستم جنى و كريز \\
\hline 1 & $\cdot \pi \cdot \Delta^{*}$ &.$/ 1 \mathrm{r}^{\circ}$ & $-e+\cdot r$ & $.1 .+1$ & سيستم قعال ساز \\
\hline
\end{tabular}

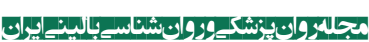

جدول ץ. آزمونهاى بايايى مدل

\begin{tabular}{|c|c|c|c|c|c|}
\hline قشدت درد & سيستم جنت/ كريز & سيستم فعال ساز & سيستم بازدارى & ناكويى هيجانى & أزمون هاى هايايى/ متغيرها \\
\hline $1 / \ldots$ & $1 / \ldots$ & .814 & . $/ \Delta T^{\circ}$ & .1198 & ألفاي كرونباخ \\
\hline $1 / \ldots$ & $1 / \ldots$ & - Mar & $\cdot 11 \cdot f$ & ./199 & هايايع تركيبي \\
\hline
\end{tabular}

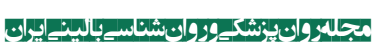

درد، هركدام يك سؤال داشتند، تمام مقادير بهدستآمده درباره

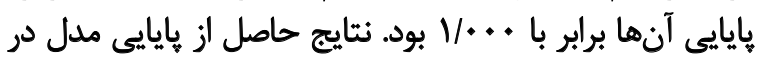

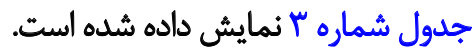

درباره روايي مدل، يس از حذف سؤالات با بارهاي عاملي كمتر

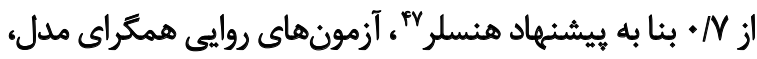

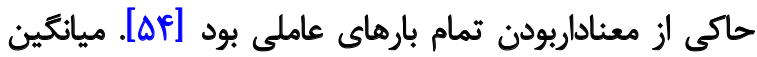

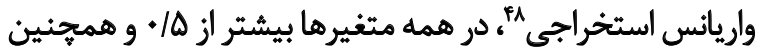

47. Henseler

48. Average Variance Extracted (AVE)
بررسى مدل در اين ثيثوهش به سبب ويزگى خاص آن (وجود

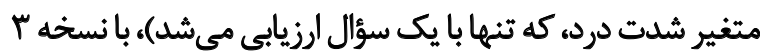

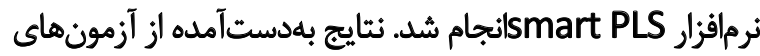

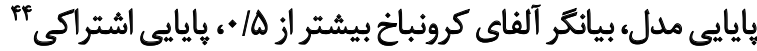

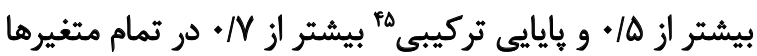

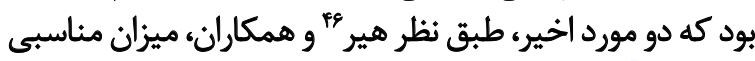

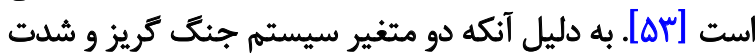

44. Comunality

45. Composit Reliability (CR)

46. Hair

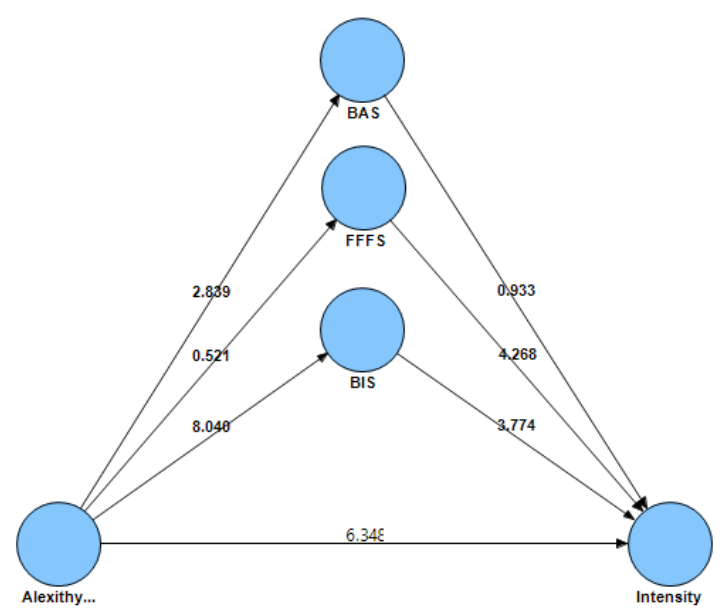


جدول f. آزمون معنادارى مسير و فرضيات يُروهش

\begin{tabular}{|c|c|c|c|c|}
\hline سطح معنادارى & خطاى استائدارد & بثا & أزمون آمارى t & مسير هاى مستقيم \\
\hline $.1 \%$ & $* H$ & $. / \sim+\bullet$ & $N \cdot r q$ & سيستم بازدارى >- ناكويى هيجانى \\
\hline .1 .1 & $.1 .+9$ &.$- / 1 f$ & T/AF" & سيستم فعال ساز >> ناكويى هيجانى \\
\hline- & .100 & -.1 .48 & $.10 T$ & سيستم جنى و كريز >- ناكوييى هيجانى \\
\hline .1 .1 & $.1 \cdot f a$ &.$/ r q$ & empa & شلت درد >- ناكويي هيجائي \\
\hline .1 .1 & $.1 \cdot 40$ &.$/ 1 V$ & $r / m e$ & شدت درد >- سيستم بازدارى رفتارى \\
\hline- & $.1 \cdot \Delta 1$ & $-.1 \cdot 4 \lambda$ &.$/ 9 \pi T$ & شدت درد >- سيستم فعال ساز رفتارى \\
\hline .1 .1 & H.HT &.$/ \Lambda$ & PREA & شدت درد >- سيستم جنى و كريز \\
\hline
\end{tabular}

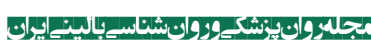

جدول ه. Z سوبل براي مسيرهاي غيرمسثقيم

\begin{tabular}{|c|c|c|c|}
\hline 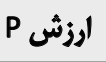 & 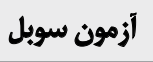 & حاصل بتاهاى مستقيه & مسير هاى غير مستقيم \\
\hline 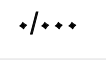 & $r / f i q$ & $.1 \cdot p q$ & نقش واسططلاى بازدارى رفتارى ميان ناكويى هيجانى و شدت درد \\
\hline.$/$ MVa & - INAS &.$- / \cdot 14$ & نقش واسطالى فعال سازى رفتارى ميان ناكويى هيجانى و شُدت درد \\
\hline$\cdot 18 \cdot 0$ &.$|\Delta| \&$ & . $/ \Delta r$ & ثقش واسطهاي جنى و كريز ميان ناكويى هيجانى و شدت درد \\
\hline
\end{tabular}

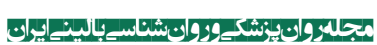

بر ميزان شدت درد ندارد. از طرف ديكر، ناكويى هيجانى مي تواند

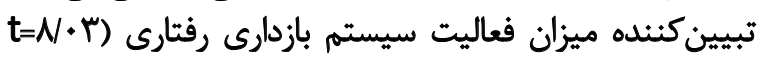

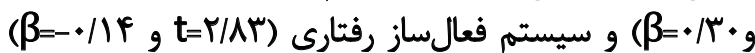

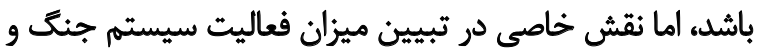

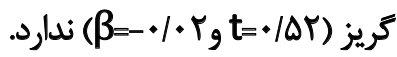

براى تعيين نقش واسطهاى سيستمهاى مغزى رفتارى ميان

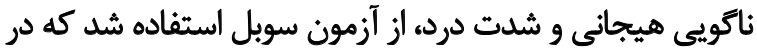

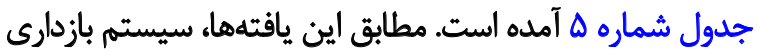

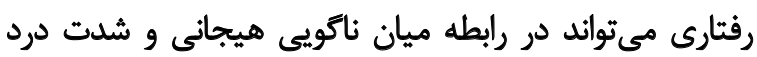

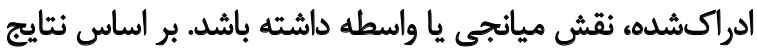

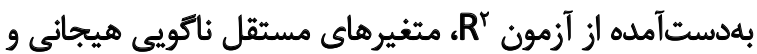

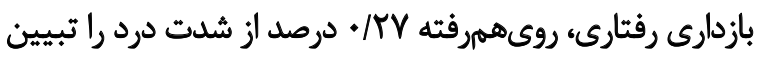

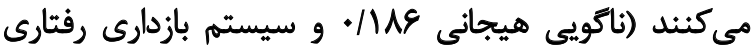

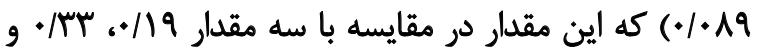
19V

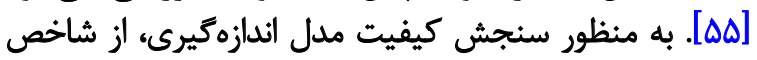

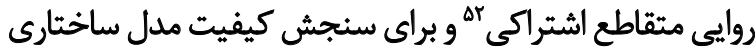

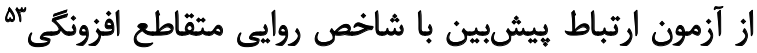

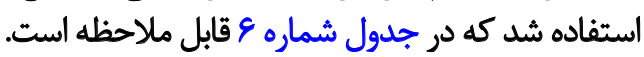

51. Chin

52. Cross Validity Comunality (CV. Com)

53. Cross Validity Redundancy (CV. Red)
كمتر از ميزان بايايى تركيبى بود. درباره روايى واكراه آزمون

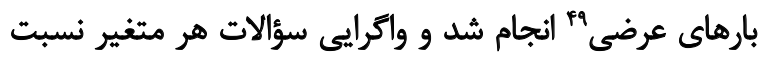

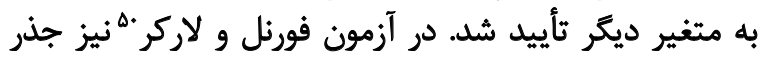

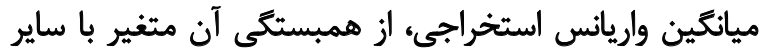
متغيرها بيشتر بود.

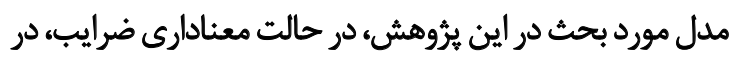

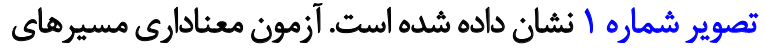

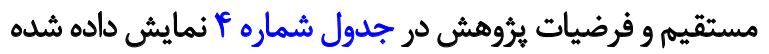

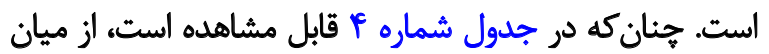

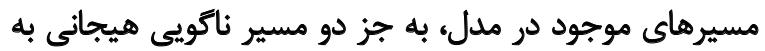

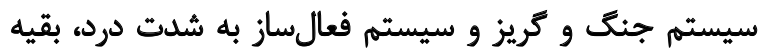

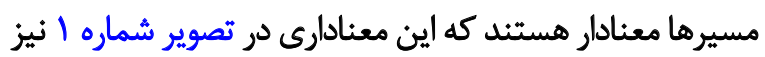
مشاهده مي شود.

نتايج حاصل از آزمون معنادارى مدل، بيانكر اين موارد

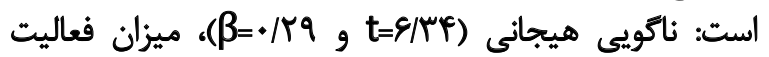

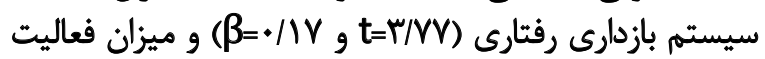

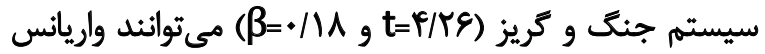

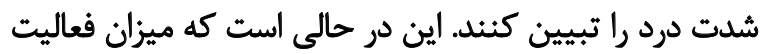

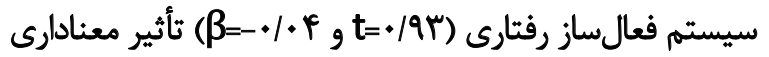

49. Cross loading

50. Fornell \& Larcker 
جدول و. آزمونهاي سنجش كيفيت مدل

\begin{tabular}{|c|c|c|c|c|c|}
\hline جنكَ و مريز & قعال ساز رفتارى & بازمارى رقتارى & نائويى هيجانى & 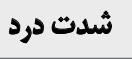 & أزمونهاى سنجش كيفيت مدل / مثغيرها \\
\hline$\%$ &.$/ 1 W$ &.$/ \circ A V$ & 年 &.$- / \ldots$ & كيفيت مدل اندازٔكيرى (شاخص CV.Com) \\
\hline$-.1 . .1$ & $1 . .4$ &.$\% \Delta V$ & . & .1198 & كيفيت مدل ساختارى (شاخص CV. Red) \\
\hline
\end{tabular}

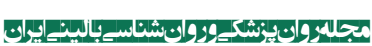

محرك هيجانى در سيستم ليمبيك كاهش مى يابد و در مقابل،

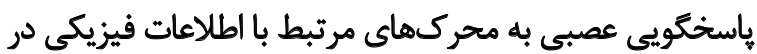

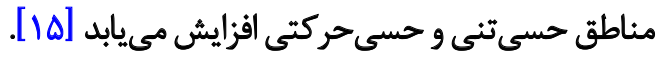
اين احتمال وجود دارد كه نقص در توانايى نمادىسازى

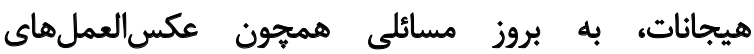
فيزيولوزيكى غيرعادى و توانايى آسيبديده براي خودئي

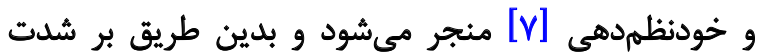

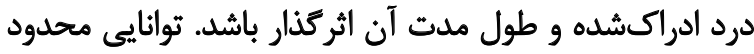

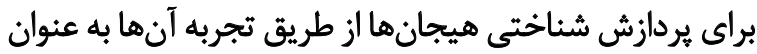

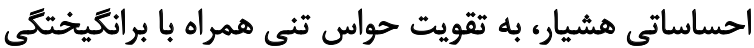

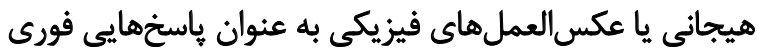

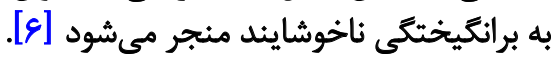

نتايج اين يُروهش نشان داد سيسته بازدارى رفتارى، ميان

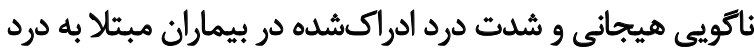

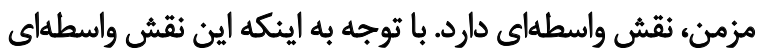

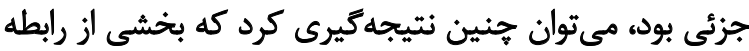

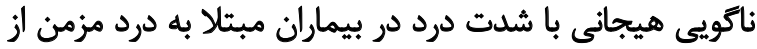

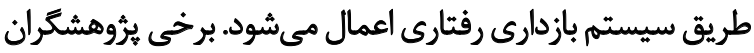

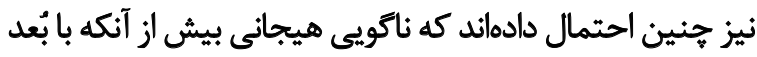

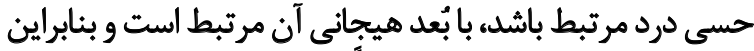

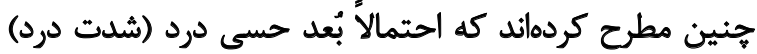

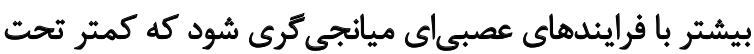
تأثير عواطف قرار دارند.

اين احتمال وجود دارد كه سازمان نورونى در آلكسيتايميا

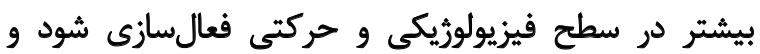

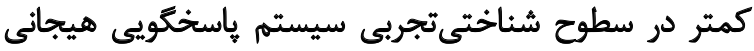

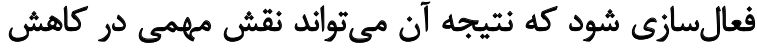

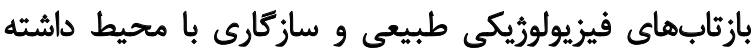

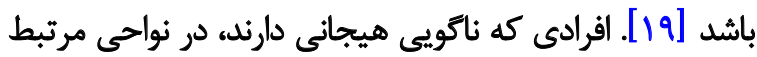

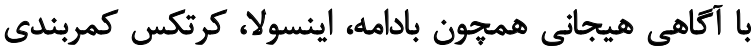

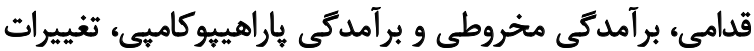

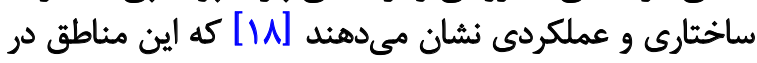

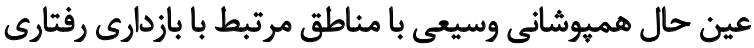

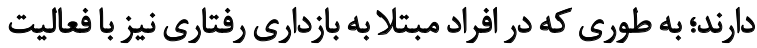

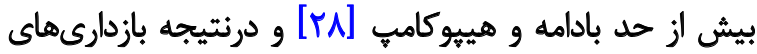

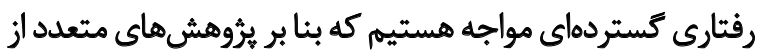

مقادير بهدستآمده از شاخص روايى متقاطع اشتراكى، در

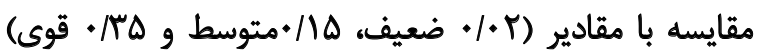

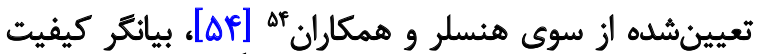

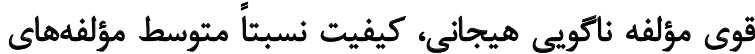

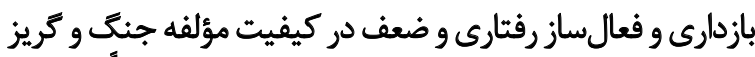

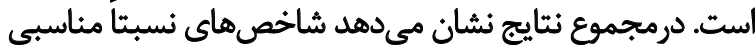

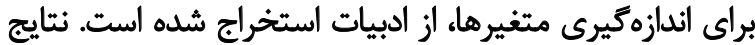

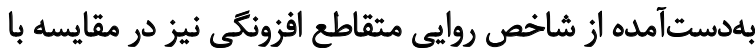

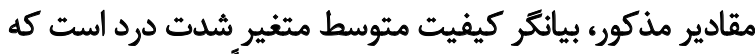

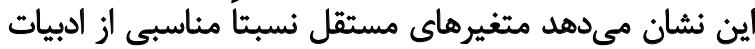

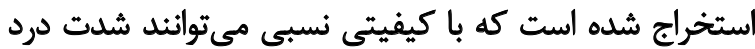

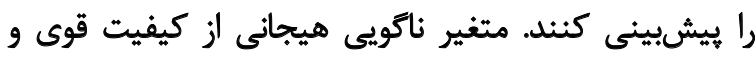

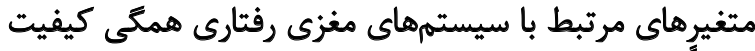

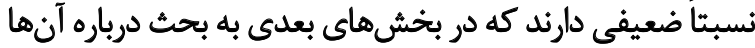

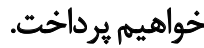

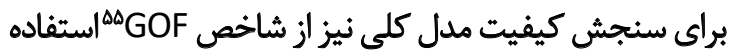

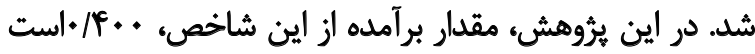

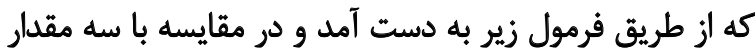

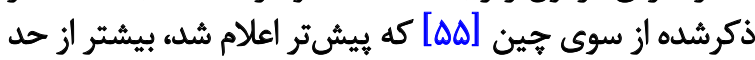
متوسط ارزيابى مى سىود.

GOF $=$ Vavrage(Comunalitie) ${ }^{*} R^{\wedge} 2=0.400$

?

يافتههاى اين بروهش نشان داد رابطه ناتويى هيجانى با شدت

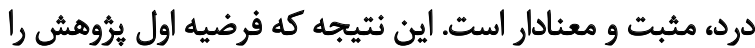

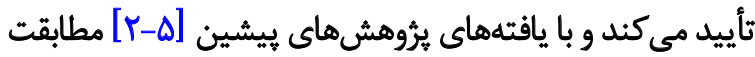

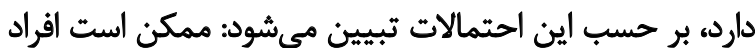

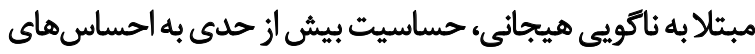

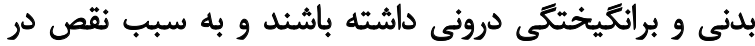

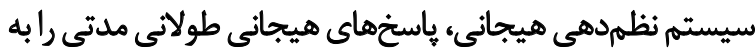

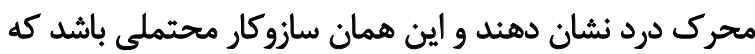

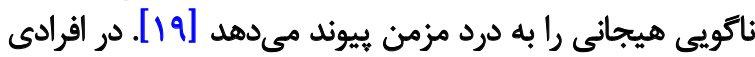

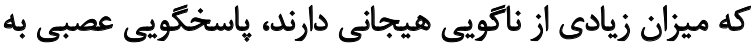

54. Henseler

55. Goodness of Fit (GOF) 


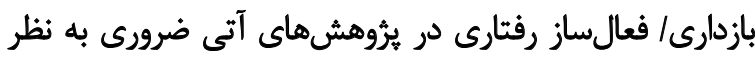

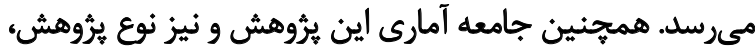

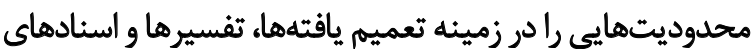

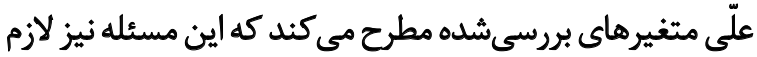

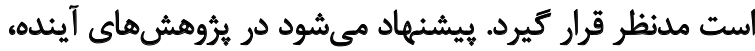

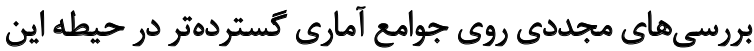

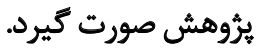

ملاحظات اخلاقى

\section{ييروى از اصول اخلالق يؤوهش}

در اين ثروهش بيماران فرم رعايت نكات اخلاقي را تكميل و

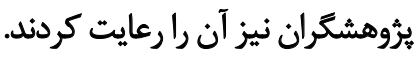

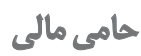

اين مقاله حامى مالى ندارد.

$$
\text { مشاركت نويسند تكان }
$$

تمام نويسندكان در آمادهسازى اين مقاله مشاركت داشتهاند.

$$
\text { تعارض مثاقع }
$$

بنابر اظهار نويسندكان، اين مقاله تعارض منافع ندارد.

$$
\text { سياسكَّزاوى }
$$

در يايان از همكارى كلينيك درد ماهان، كلينيك جامع طب

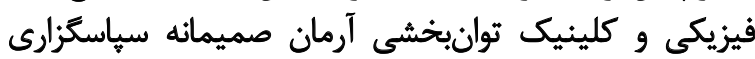
مئيمن فيني
مشخصههاى رفتارى اساسى در ميان بيماران مبتّلا به دردهاى

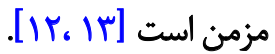

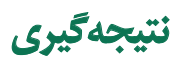

ناكويى هيجانى به معناي ناتوانى در شناسايى و توصيف عواطف

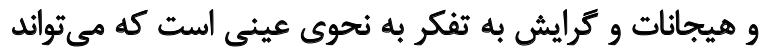

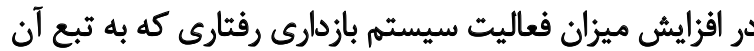

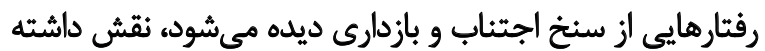

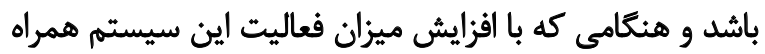

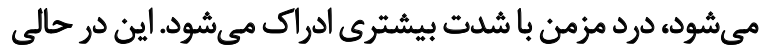

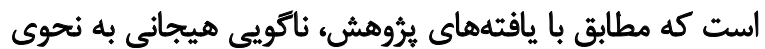

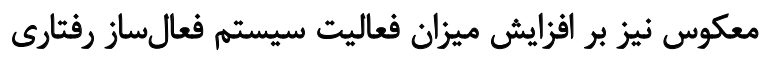

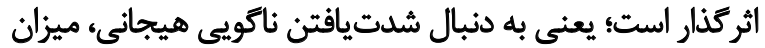

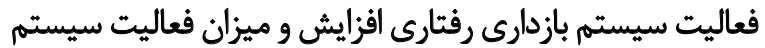

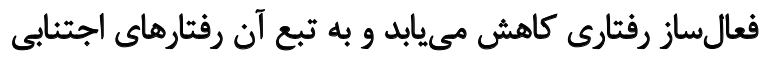

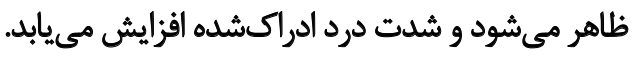

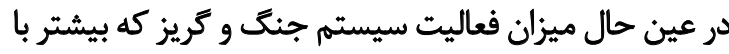

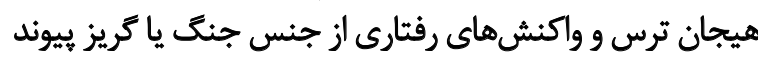

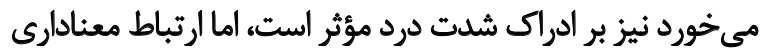

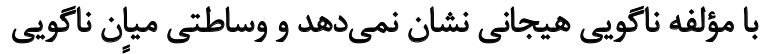

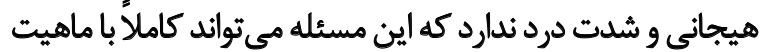

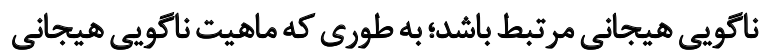

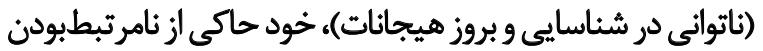

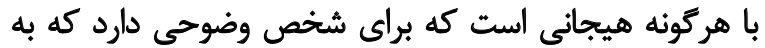
سبب آن واكنشهائه از سنخ جنك يا كريز را بركزيده است.

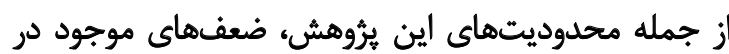

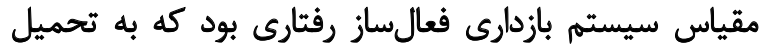

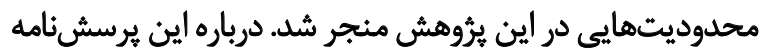

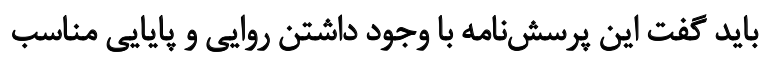

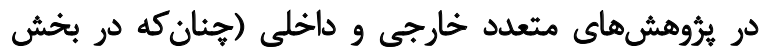

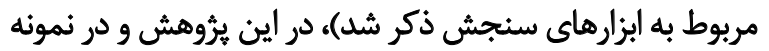

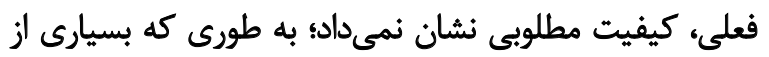

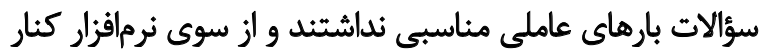

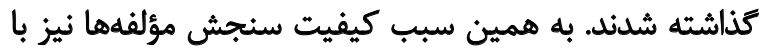

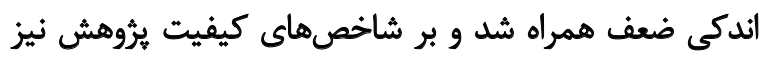

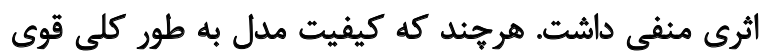

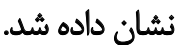

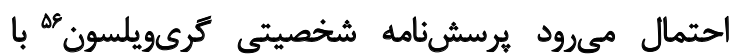

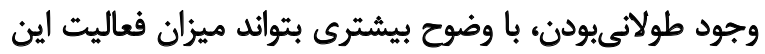

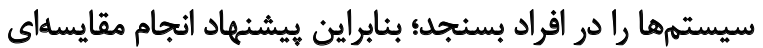

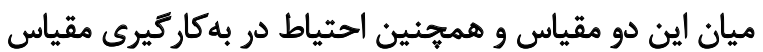

56. Gray-Wilson Personality Questionaire (GWPQ) 


\section{References}

[1] Reid KJ, Harker J, Bala MM, Truyers C, Kellen E, Bekkering GE, et al. Epidemiology of chronic non-cancer pain in Europe: Narrative review of prevalence, pain treatments and pain impact. Current Medical Research and Opinion. 2011; 27(2):449-62. [DOI:10.1 185/03007995.2010.545813]

[2] Shibata M, Ninomiya T, Jensen MP, Anno K, Yonemoto K, Makino $\mathrm{S}$, et al. Alexithymia is associated with greater risk of chronic pain and negative affect and with lower life satisfaction in a general population: The hisayama study. PLOS One; 9(3):e90984. [DOI:10.1371/journal.pone.0090984]

[3] Bottiroli S, Galli F, Viana M, Sances G, Allena M, Ghiotto N, et al. Alexithymia and chronic migraine with medication overuse: what relationship. The Journal of Headache and Pain. 2015; 16(Suppl. 1):A150.[DOI:10.1186/1129-2377-16-S1-A150]

[4] Burger AJ, Lumley MA, Carty JN, Latsch DV, Thakur ER, HydeNolan ME, et al. The effects of a novel psychological attribution and emotional awareness and expression therapy for chronic musculoskeletal pain: A preliminary, uncontrolled trial. Journal of Psychosomatic Research. 2016; 81:1-8. [DOI:10.1016/j.jpsychores.2015.12.003]

[5] Chang MC, Chen PF, Lung FW. Personality disparity in chronic regional and widespread pain. Psychiatry Research. 2017; 254:284-9. [DOI:10.1016/j.psychres.2017.04.059]

[6] Taylor GJ, Bagby RM, Parker JD. The alexithymia construct. A potential paradigm for psychosomatic medicine. Psychosomatics. 1991; 32(2):153-64. [DOI:10.1016/S0033-3182(91)72086-0]

[7] Nemiah JC, Sifneos PE. Affects and fantasy in patients with psychosomatic disorders. In Hill O, editor. Modern Trends in Psychosomatic Medicine. London: Lexis Nexis Butterworths; 1970.

[8] Sifneos PE. The prevalence of 'alexithymic' characteristics in psychosomatic patients. Psychotherapy and Psychosomatics. 1973; 22(2-6):255-62. [DOI:10.1159/000286529] [PMID]

[9] Melin EO, Thulesius HO, Persson BA. Affect school for chronic benign pain patients showed improved alexithymia assessments with TAS-20. BioPsychoSocial Medicine. 2010; 4(5):1-10. [DOI:10.1186/1751-0759-4-5]

[10] Davydov DM, Luminet O, Zech E. An externally oriented style of thinking as a moderator of responses to affective films in women. International Journal of Psychophysiology. 2013; 87(2):152-64. [DOI:10.1016/j.ijpsycho.2012.12.003]

[11] Mc Craken LM, Samuel VM. The role of avoidance, pacing, and other activity patterns in chronic pain. Pain. 2007; 130(2):119-25. [DOI:10.1016/j.pain.2006.11.016]

[12] Trost Z, France CRS, Tomas JS. Pain-related fear and avoidance of physical exertion following delayed-onset muscle soreness. Pain. 2011; 152(7):1540-7. [DOI:10.1016/j.pain.2011.02.038]

[13] Volders S, Boddez Y, De Peuter S, Meulders A, Vlaey JWS. Avoidance behavior in chronic pain research: A cold case revisited. Behaviour Research and Therapy. 2015; 64:31-7. [DOI:10.1016/j.brat.2014.11.003]

[14] Pouga L, Berthoz S, de Gelder B, Gre`zes J. Individual differences in socioaffective skills influence the neural bases of fear processing: the case of alexithymia. Human Brain Mapping. 2010; 31(10):1469-81. [DOI:10.1002/hbm.20953]
[15] Reker M, Ohrmann P, Rauch AV, Kugel H, Bauer J, Dannlowski $U$, et al. Individual differences in alexithymia and brain response to masked emotion faces. Cortex. 2010; 46(5):658-67. [DOI:10.1016/j.cortex.2009.05.008]

[16] Ihme K, Dannlowski U, Lichev V, Stuhrmann A, Grotegerd D, Rosenberg N, et al. Alexithymia is related to differences in gray matter volume: A voxel-based morphometry study. Brain Research. 2013; 1491:60-7. [DOI:10.1016/j.brainres.2012.10.044]

[17] Grabe HJ, Wittfeld K, Hegenscheid K, Hosten N, Lotze M, Janowitz D, et al. Alexithymia and brain gray matter volumes in a general population sample. Human Brain Mapping. 2014; 35(12):5932-45. [DOI:10.1002/hbm.22595]

[18] Laricchiuta D, Petrosini L, Picerni E, Cutuli D, Iorio M, Chiapponi $\mathrm{C}$, et al. The embodied emotion in cerebellum: A neuroimaging study of alexithymia. Brain Structure and Function. 2015 220(4):2275-87. [DOI:10.1007/s00429-014-0790-0]

[19] Kano M, Fukudo S. The alexithymic brain: The neural pathways linking alexithymia to physical disorders. BioPsychoSocial Medicine. 2013; 7(1):1. [DOI:10.1186/1751-0759-7-1] [PMID] [PMCID]

[20] Barros-loscertales A, Meseguer V, Sanjuan A, Belloch V, Parcet MA, Torrubia R, et al. Behavioral inhibition system activity is associated with increased amygdale and hippocampal gray matter volume: A Voxel-based morphometry study. Neuroimage. 2006; 33(3):1011-5. [DOI:10.1016/j.neuroimage.2006.07.025]

[21] Hahn t, Dresler t, Plichta MM, Ehlis AC, Ernst LH, Markulin $\mathrm{F}$, et al. Functional amydala-hippo campus connectivity during anticipation of aversive events is associated with grays trait "sensitivity to punishment". Biological Psychiatry. 2010; 68(5):459-64. [DOI:10.1016/j.biopsych.2010.04.033]

[22] Gray JA. Oxford psychology series. The neuropsychology of anxiety: An enquiry into the functions of the septo-hippocampal system. Oxford: Oxford University Press; 1982.

[23] Corr PJ. Reinforcement sensitivity theory and personality. Neuroscience \& Biobehavioral Reviews. 2004; 28(3):317-32 [DOI:10.1016/j.neubiorev.2004.01.005]

[24] Hahn T, Dresler T, Ehlis AC, Pyka M, Dieler AC, Saathoff C, et al. Randomness of resting-state brain oscillations encodes Grays Personality trait. NeuroImage. 2012; 59(2):1842-5. [DOI:10.1016/j. neuroimage.2011.08.042]

[25] Avila C, Garbin G, Sanjuan A, Forn C, Barros Loscer tales A Bustamants J C, et al. Frontostriatal response to set switching is moderated by reward sensitivity. Social Cognitive and Affective Neuroscience. 2011; 7(4):423-30. [DOI:10.1093/scan/nsr028]

[26] Kimbrel NA, Nelson Gray RO, Mitchell JT. BIS, BAS, and Bias: The role of personality and cognitive bias in social anxiety. Personality and Individual Differences. 2012; 52(3):395-400. [DOI:10.1016/j.paid.2011.10.041]

[27] Poythress NG, Hall JR. Psychopathy and impulsivity reconsidered. Aggression and Violent Behavior. 2011; 16(2):120-34. [DOI:10.1016/j.avb.2011.02.003]

[28] Corr P. Anxiety: Splitting the phenomenological atom Personality and Individual Differences. 2011; 50(7):889-97. [DOI:10.1016/j.paid.2010.09.013]

[29] Salaffi F, Stancati A, Silvestri CA, Ciapetti A, Grassi W. Minimal clinically important changes in chronic musculoskeletal pain 
intensity measured on a numerical rating scale. European Journal of Pain. 2004; 8(4):283-91. [DOI:10.1016/j.ejpain.2003.09.004]

[30] Breivik H. Patients' subjective acute pain rating scales (VAS, NRS) are fine; more elaborate evaluations needed for chronic pain, especially in the elderly and demented patients. Scandinavian Journal of Pain. 2017; 15(1):73-4. [DOI:10.1016/j.sjpain.2016.12.007]

[31] Zalmay P, Williams AC. How do medical students use and understand painrating scales. Scandinavian Journal of Pain. 2017; 15(1):68-72. [DOI:10.1016/j.spain.2016.12.007]

[32] Ferraz MB, Quaresma MR, Aquino LRL, Atra E, Tugwell P, Goldmith $\mathrm{CH}$. Reliability of pain scales in the assessment of literate and illiterate patients with rheumatoid arthritis. Journal of Rheumatology. 1990; 17(8):1022-4. [PMID]

[33] Hawker GA, Mian S, Kendzerska T, French M. Measures of adult pain: Visual Analog Scale for Pain (VAS Pain), Numeric Rating Scale for Pain (NRS Pain), McGill Pain Questionnaire (MPQ), Short-Form McGill Pain Questionnaire (SF-MPQ), Chronic Pain Grade Scale (CPGS), Short Form-36 Bodily Pain Scale (SF36 BPS), and measure of Intermittent and Constant Osteoarthritis Pain (ICOAP). Arthritis Care \& Research. 2011, 63(S11):S240-52. [DOI:10.1002/acr.20543]

[34] Phan NQ, Blome C, Fritz F, Gerss J, Reich A, Ebata T, et al. Assessment of pruritus intensity: Prospective study on validity and reliability of the visual analogue scale, numerical rating scale and verbal rating scale in 471 patients with chronic pruritus. Acta Dermato-Venereologica. 2012; 92(5):502-7. [DOI:10.2340/000155551246] [PMID]

[35] Carver CS, White TL. Behavioral inhibition, behavioral activation, and affective responses to impending verard and punishment: The BIS/BAS scales. Journal of Personality and Social Psychology. 1994; 67(2):319-33. [DOI:10.1037/0022-3514.67.2.319]

[36] Ross SR, Millis SR, Bonebright TL, Bailley SE. Confiromatory factor analysis of the behavioral inhibition and activation scales. Personality and Individual Differences. 2002; 33(6):861-65. [DOI:10.1016/S0191-8869(01)00196-9]

[37] Campbell-Sills L, Liverant GI, Brown TA. Psychometric evaluation of behavioral inhibition/ behavioral activation scales in a large sample of outpatients with anxiety and mood disorders. Psychological Assessment. 2004;16(3):244-54. [DOI:10.1037/10403590.16.3.244]

[38] Muller JM, Wytykowska AM. Psychometric properties and validation of polish adaptation of carver and whites BIS/BAS scales. Personality and Individual Differences. 2005; 39(4):795805. [DOI:10.1016/j.paid.2005.03.006]

[39] Gray JD, Hanna D, Gillen A, Rushe T. A closer look at Carver andWhite's BIS/BAS scales: Factor analysis and age group differences. Personality and Individual Differences. 2016; 95:20-4. [DOI:10.1016/j.paid.2016.02.022]

[40] Mohammadi N. [The psychometric properties of the Behavioral Inhibition System (BIS) and Behavioral Activation System (BAS) scales among students of Shiraz University (Persian)]. Clinical Psychology \& Personality. 2008; 1(28):61-8.

[41] Abdollahi Majarshin R, Bakhshipoor A, Mahmood Aliloo M. [Reliability and validity of the Behavioral Inhibition and Activation Systems (BIS/BAS) scale in student population of Tabriz University (Persian)]. Journal of Advanced Psychological Research. 2012, 7(28):123-39.
[42] Amiri S, Hasani J. [Evaluation of psychometric properties of Behavioral Activation System Scale (BAS) and Behavioral Inhibition System (BIS) related to impulsivity and anxiety (Persian)]. Razi Medical Journal. 2016, 23(144):68-80

[43] Heym N, Ferguson E, Lawrence C. An evaluation of the relationship between Gray's revised RST and Eysenck's PEN: Distinguishing BIS and FFFS in carver and white's BIS/BAS scales. Personality and Individual Differences. 2008; 45(8):709-15. [DOI:10.1016/j.paid.2008.07.013]

[44] Bagby RM, Parker JDA, Taylor GJ. The twenty-item Toronto Alexithymia scale-I. Item selection and cross-validation of the factor structure. Journal of Psycho Somatic Research. 1994; 38(1):2332. [DOI:10.1016/0022-3999(94)90005-1]

[45] Bagby RM, Taylor GJ, Parker JDA. The twenty-item Toronto Alexithymia scale-II. Convergent, discriminant, and concurrent validity. Journal of Psychosomatic Research. 1994; 38(1):33-40. [DOI:10.1016/0022-3999(94)90006-X]

[46] Parker JDA, Taylor GJ, Bagby RM. The relationship between emotional intelligence and alexithymia. Personality and Individual Differences. 2011; 30(2001):107-15. [DOI:10.1016/S01918869(00)00014-3]

[47] Parker JDA, Taylor GJ, Bagby RM. The 20-item Toronto Alexithymia scale: III reliability and factorial validity in a community population. Journal of Psychosomatic Research. 2003; 55(3):26975. [DOI:10.1016/S0022-3999(02)00578-0]

[48] Palmer BR, Gignac G, Manocha R, Syough C. A psychometric evaluation of the Mayer-Salovey-Caruso Emotional Intelligence Test version 2.0. Intelligence. 2005; 33(3):285-305. [DOI:10.1016/j. intell.2004.11.003]

[49] Pandey R, Mandal MK, Taylor GJ, Parker JDA. Cross-cultural alexithymia: Development and validation of a Hindi translation of the 20-item Toronto Alexithymia scale. Journal of Clinical Psychology. 1996; 52(2):173-6. [DOI:10.1002/(SICI)10974679(199603)52:23.0.CO;2-V]

[50] Taylor GJ, Bagby RM. An overview of the alexithymia construct. In Bar-On R, Parker JDA, editors. The Handbook of Emotional Intelligence: Theory, Development, Assessment, and Application at Home, School, and in the Workplace. San Francisco: Jossey-Bass; 2000

[51] Besharat MA. Reliability and factorial validity of Farsi version of the Toronto Alexithymia Scale with a sample of Iranian students. Psychological Reports. 2007; 101(1):209-20. [DOI:10.2466/ pr0.101.1.209-220

[52] Besharat MA. [Toronto Alexithymia scale: Questionnaire, method of implementation and scoring (Persian)]. Journal of Developmental Psychology. 2013, 10(37):90-2

[53] Hair GF, Sarstedt M, Ringle CM, Mena JA. An assessment of the use of partial least squares structural equation modeling in marketing research. Journal of Academy of Marketing Science. 2012; 40(3):414-33. [DOI:10.1007/s11747-011-0261-6]

[54] Henseler J, Ringle CM, Sinkovics RR. The use of partial least squares path modeling in international marketing. In: Sinkovics RR, Ghauri PN, editors. Advances in International Marketing. Emerald Bingley; 2009. [DOI:10.1108/S1474-7979(2009)0000020014]

[55] Chin WW. Issues and opinion on structural equation modeling Management Information Systems Quarterly. 1998; 21(1):7-16. 